\title{
КРЕДИТНЫЕ ГАРАНТИИ ДЛЯ МАЛЫХ И СРЕДНИХ ПРЕДПРИЯТИЙ: ЗАРУБЕЖНЫЙ ОПЫТ
}

\author{
Пиньковецкая Юлия Семеновна, \\ judy54@yandex.ru \\ Ульяновский государственный университет, \\ Россия, 432000, г. Ульяновск, ул. Л. Толстого, 42
}

\begin{abstract}
Пиньковецкая Юлия Семеновна, кандидат экономических наук, доцент кафедры экономического анализа и государственного управления Ульяновского государственного университета.
\end{abstract}

\begin{abstract}
Актуальность исследования обусловлена тем, что малое и среднее предпринимательство играет важную роль в национальных экономиках абсолютного большинства развитых и развивающихся стран. Вместе с тем одним из существенных барьеров на пути его дальнейшего роста является слабое кредитование банками предпринимательского сектора. Одним из главных направлений преодоления сложившейся ситуации является разработка и реализация программ гарантирования кредитов. Цель: изучение опыта кредитования малых и средних предприятий с использованием гарантийных фондов, анализ принципов и конщепций создания систем кредитных гарантий. В процессе работы были рассмотрены проблемы выдачи банковских кредитов малому и среднему бизнесу, изучен международный опыт реализации программ гарантийного кредитования, рассмотрены сложившиеся схемы организации этой деятельности. Методология основывается на анализе деятельности национальных, региональных и отраслевых гарантийных фондов, а также финансово-кредитных организаций, специализирующихся на выдаче гарантий для малых и средних предприятий. При этом основное внимание уделяется государственным гарантиям кредитования таких предприятий. Рассматривается опыт деятельности систем кредитных гарантий в Европе, Северной и Латинской Америке, Азии и Африке. Результаты показали, что программы гарантирования кредитов играют большую роль в снижении ограничений на банковское финансирование для малых и средних предприятий. В статье приведены основные принципы и концепции реализации гарантийных программ, осуществляемых с государственным участием. Показано, что кредитные гарантии являются одним из инструментов, широко используемых при поддержке предпринимательского сектора экономики. При этом наиболее целесообразным является выдача гарантий, обеспечивающих частичный объем кредитования, когда заемщики также несут определенную ответственность по возврату полученных средств. Результаты исследования могут быть использованы в деятельности гарантийных организаций, финансово-кредитных учреждений, органов государственной власти. Итоги анализа представляют интерес для руководителей и работников малых и средних предприятий.
\end{abstract}

Ключевые слова: Малые и средние предприятия, гарантийные фонды, банки, системы кредитных гарантий, финансирование предпринимательства.

\section{Введение}

Малые и средние предприятия (МСП) в большинстве современных стран рассматриваются в качестве основы экономики. Они, как показывают исследования, обеспечивают значительный экономический рост, участвуют во внедрении инноваций, снижают безработицу за счет организации новых рабочих мест и создают социальную стабильность [1, 2, 3]. В странах Европейского Союза доля МСП достигает 99,8 \% от всех предприятий, почти 60 \% добавленной стоимости и почти 70 \% общей численности рабочей силы [4]. В странах Азии по данным исследования [5] средние значения вклада МСП в 
экономику также были достаточно весомы. На них приходилось 98 \% всех предприятий, 38 \% валового внутреннего продукта и $66 \%$ национальной рабочей силы. В среднем доля МСП в экспорте по азиатским странам достигала $30 \%$ от общего объема. Значимость МСП наиболее существенна в развивающихся странах, в которых сравнительно мало крупных предприятий. Поэтому в этих странах МСП оказывают решающее влияние на социально-экономическое развитие за счет сокращения уровня безработицы и снижения количества бедных людей [6].

В силу большого значения предпринимательского сектора экономики представляется актуальным обеспечение МСП стабильным и доступным финансированием. Вместе с тем накопленный опыт показывает, что такие предприятия сталкиваются с серьезными проблемами при привлечении необходимых средств. Как показывают научные исследования $[7,8]$, многие МСП не имеют доступа к банковскому финансированию даже под высокие проценты, что негативно влияет на их экономические показатели.

Для решения проблемы устойчивого финансирования МСП как в развитых, так и в развивающихся странах начиная со второй половины XX в. в Европе и США, а немного позже в Азии и Африке, были созданы специализированные системы, обеспечивающие кредитные гарантии при участии правительств и региональных властей [9]. В 1990-е годы кредитные гарантийные системы были созданы в странах с переходной экономикой Восточной Европы и бывших республик Советского Союза. В начале 2000-х гг. более 170 стран использовали разнообразные программы кредитных гарантий [10]. По мнению многих экспертов, такие программы способны обеспечить сокращение разрыва между потребностями МСП в кредитах и соответствующими предложениями финансовых структур $[11,12]$. Гарантом выступают, как правило, специализированные организации, управляемые правительствами, региональными или отраслевыми органами, которые стараются облегчить доступ к финансированию, предоставляя кредиторам комфортную гарантию на значительную часть долга [13]. Кредитные гарантии применяются во многих странах при привлечении денежных средств в приоритетные сегменты экономики. В результате многие МСП с экономически жизнеспособными проектами получают необходимое финансирование. Рост числа поддерживаемых государством гарантийных фондов подтвердил, что системы гарантий особенно важны в разгар рецессии, когда они способны стимулировать рост МСП за счет снижения их внешних затрат на финансирование [14]. Исследование, проведенное по инициативе Организации экономического сотрудничества и развития показало, что кредитные гарантии широко используются в развитых странах в качестве важных инструментов для смягчения финансовых ограничений для МСП и стартапов [15].

Наиболее крупные системы кредитных гарантий, которые в настоящее время показали свою высокую эффективность, расположены во многих разных странах, находящихся в Северной и Латинской Америке, Азии и Европе. Среди них можно выделить такие организации: в США - «7(a) Loan Program», в Бразилии - «FAMPE», в Чили «FOGAPE», в Индии - «CGTMSE», в Южной Kopee - «Korea Technology Finance Corporation (KIBO)», в Японии - «Japanese Credit Guarantee Corporation (CGC)», в Португалии - «IAPMEI», в Италии - «Confidi», в Испании - «CERSA», во Франции «SOCAMA», в Германии - «Bürgschaftsbanken», в Финляндии - «Finnvera». Кроме того, в странах Европейского Союза кроме национальных гарантийных фондов существует также общеевропейская система - «European Commission's SME Guarantee Facility» [15]. Абсолютное большинство из них действуют по сходным схемам, предоставляя уполномоченным банкам гарантии для выдачи кредитов субъектам малого и среднего предпринимательства. Как и в России, деятельность этих кредитных систем поддерживается и 
финансируется национальными Правительствами, а в Европейском Союзе -соответствующими финансовыми органами.

Целью данного исследования является изучение опыта кредитования МСП с использованием гарантийных фондов, анализ принципов и концепций создания систем кредитных гарантий. В процессе работы были решены следующие задачи:

- рассмотрены проблемы выдачи банковских кредитов малому и среднему бизнесу;

- изучен международный опыт реализации программ гарантийного кредитования;

- рассмотрены сложившиеся схемы организации этой деятельности.

В настоящей статье мы представили обзор наиболее важных, на наш взгляд, научных исследований, посвященных проблемам кредитных гарантий. Обзор охватывает работы исследователей, выполненные с 1990-х гг. по настоящее время. При этом мы постарались ответить на вопросы об основных аспектах создания, о роли систем гарантирования кредитов в рыночных национальных экономиках, экономических, организационных и управленческих аспектах их деятельности.

\section{Обзор литературы}

Существует большое количество научных исследований, рассматривающих теоретические и прикладные проблемы кредитных гарантий в различных странах.

Краткая характеристика наиболее важных из них приведена в таблице от более ранних к более поздним публикациям.

таблица. Наиболее важные зарубежные научные работы по системам гарантирования кредитов

Table. Most important foreign works on systems credits guarantee

\begin{tabular}{|c|c|c|}
\hline $\begin{array}{c}\text { Автор (организация) и год } \\
\text { издания } \\
\text { Author (organization) and } \\
\text { year of publication }\end{array}$ & $\begin{array}{l}\text { Объект исследования } \\
\text { Research object }\end{array}$ & $\begin{array}{l}\text { Основные выводы } \\
\text { Main conclusions }\end{array}$ \\
\hline $\begin{array}{l}\text { Deelen L., Molenaar K., } \\
2004 \text { [16] }\end{array}$ & $\begin{array}{l}\text { Системы кредитных гарантий } \\
\text { в различных странах } \\
\text { Credit guarantee systems in vari- } \\
\text { ous countries }\end{array}$ & $\begin{array}{l}\text { Предложены мероприятия по обеспечению } \\
\text { работы гарантийных фондов } \\
\text { Measures to ensure the operation of guarantee } \\
\text { funds were proposed }\end{array}$ \\
\hline Riding, А. и др., 2007 [17] & $\begin{array}{l}\text { Канадская система гарантиро- } \\
\text { вания займов } \\
\text { Canadian Loan Guarantee } \\
\text { System }\end{array}$ & $\begin{array}{l}\text { Показано, что МСП не получили бы кредиты } \\
\text { на общих условиях без гарантий } \\
\text { It is shown that small and medium-sized enter- } \\
\text { prises would not receive loans on general terms } \\
\text { without guarantees }\end{array}$ \\
\hline Beck Т. и др., 2008 [18] & $\begin{array}{l}76 \text { фондов в разных странах } \\
76 \text { funds in different countries }\end{array}$ & $\begin{array}{l}\text { Предложена типология фондов гарантирова- } \\
\text { ния кредитов } \\
\text { A typology of loan guarantee funds is proposed }\end{array}$ \\
\hline $\begin{array}{l}\text { Posey R., Reichert A., } 2011 \\
{[19]}\end{array}$ & $\begin{array}{l}4240 \text { фирм, получивших кре- } \\
\text { дитные гарантии в США } \\
4,240 \text { firms that received loan } \\
\text { guarantees in the United States }\end{array}$ & $\begin{array}{l}\text { Наличие гарантий обеспечивает более низкие } \\
\text { процентные ставки по кредитам } \\
\text { Availability of guarantees ensures lower interest } \\
\text { rates on loans }\end{array}$ \\
\hline Saadani Y. и др., 2011 [20] & $\begin{array}{l}\text { Системы кредитных гарантий } \\
\text { в странах Ближнего Востока и } \\
\text { Северной Африки } \\
\text { Credit guarantee systems in the } \\
\text { Middle East and North Africa }\end{array}$ & $\begin{array}{l}\text { Возможно наращивание гарантий для микро- } \\
\text { предприятий, которые получают их в недо- } \\
\text { статочном количестве } \\
\text { It is possible to build up guarantees for micro- } \\
\text { enterprises that do not receive them enough }\end{array}$ \\
\hline
\end{tabular}




\begin{tabular}{|c|c|c|}
\hline Leone Р. и др., 2013 [21] & $\begin{array}{l}\text { Консорциум кредитных га- } \\
\text { рантий в Италии } \\
\text { Credit Guarantee Consortium in } \\
\text { Italy }\end{array}$ & $\begin{array}{l}\text { Подтверждена высокая эффективность дея- } \\
\text { тельности консорциума } \\
\text { High efficiency of the consortium has been } \\
\text { confirmed }\end{array}$ \\
\hline Gropp, R. и др., 2013 [22] & $\begin{array}{l}\text { Банки Германии } \\
\text { Banks in Germany }\end{array}$ & $\begin{array}{l}\text { Использование гарантий обеспечивает сни- } \\
\text { жение процентной ставки, продление срока } \\
\text { погашения кредита и более низкие требова- } \\
\text { ния к обеспечению } \\
\text { The use of guarantees ensures lower interest } \\
\text { rates, longer loan maturities and lower collateral } \\
\text { requirements }\end{array}$ \\
\hline Anginer D., 2014 [23] & $\begin{array}{l}\text { Система кредитных гарантий } \\
\text { США } \\
\text { US credit guarantee system }\end{array}$ & $\begin{array}{l}\text { Необходимы государственные гарантии по } \\
\text { кредитам МСП } \\
\text { Government guarantees for loans to small and } \\
\text { medium-sized enterprises are required }\end{array}$ \\
\hline World Bank, 2015 [24] & $\begin{array}{l}\text { Системы кредитных гарантий } \\
\text { в различных странах } \\
\text { Credit guarantee systems in } \\
\text { various countries }\end{array}$ & $\begin{array}{l}\text { Предлагаются } 16 \text { принципов проектирования } \\
\text { систем кредитных гарантий } \\
16 \text { design principles for credit guarantee systems } \\
\text { were proposed }\end{array}$ \\
\hline $\begin{array}{l}\text { Chatzouz, М. и др., } 2017 \\
{[25]}\end{array}$ & $\begin{array}{l}18 \text { организаций кредитных га- } \\
\text { рантий в } 13 \text { странах и } 33 \text { банка } \\
\text { в } 17 \text { странах Западной Европы } \\
18 \text { credit guarantee organizations } \\
\text { in } 13 \text { countries and } 33 \text { banks in } \\
17 \text { countries of Western Europe }\end{array}$ & $\begin{array}{l}\text { Гарантирование кредитов обеспечивает об- } \\
\text { легчение финансирования МСП, особенно во } \\
\text { время финансовых кризисов } \\
\text { Loan guarantee provides easier financing for } \\
\text { small and medium enterprises, especially during } \\
\text { financial crises }\end{array}$ \\
\hline Liang L.W. и др., 2017 [26] & $\begin{array}{l}\text { Система кредитных гарантий в } \\
\text { Южной Kopee } \\
\text { Credit Guarantee System in } \\
\text { South Korea }\end{array}$ & $\begin{array}{l}\text { Кредитные гарантии повышают эффектив- } \\
\text { ность работы банков } \\
\text { Loan guarantees increase the efficiency of banks }\end{array}$ \\
\hline Balog A., 2018 [27] & $\begin{array}{l}\text { МСП, расположенные в стра- } \\
\text { нах Евросоюза } \\
\text { Small and medium enterprises } \\
\text { located in the EU countries }\end{array}$ & $\begin{array}{l}\text { Кредитные гарантии не только облегчают до- } \\
\text { ступ МСП к финансовым средствам, но со- } \\
\text { кращают рисковые активы банков } \\
\text { Credit guarantees not only make it easier for } \\
\text { small and medium-sized enterprises to access fi- } \\
\text { nance, but also reduce the risky assets of banks } \\
\end{array}$ \\
\hline $\begin{array}{l}\text { Saito K., Tsuruta, D., } 2018 \\
{[28]}\end{array}$ & $\begin{array}{l}\text { МСП в Японии } \\
\text { Small and medium enterprises in } \\
\text { Japan }\end{array}$ & $\begin{array}{l}\text { Гарантия } 80 \% \text { суммы кредита более целесо- } \\
\text { образна, чем полная }(100 \%) \\
\text { A guarantee of } 80 \% \text { of the loan amount is more } \\
\text { appropriate than a full guarantee }(100 \%)\end{array}$ \\
\hline $\begin{array}{l}\text { Brault J., Signore S., } 2019 \\
{[29]}\end{array}$ & $\begin{array}{l}360000 \text { кредитов по } 19 \text { странам } \\
\text { Евросоюза (с } 2002 \text { по } 2016 \text { гг.) } \\
360000 \text { loans across } 19 \text { EU } \\
\text { countries (from } 2002 \text { to } 2016)\end{array}$ & $\begin{array}{l}\text { Гарантированные кредиты положительно } \\
\text { влияют на рост активов фирм, объем произ- } \\
\text { водства, занятость населения и снижают ве- } \\
\text { роятность банкротства } \\
\text { Guaranteed loans have a positive effect on the } \\
\text { growth of firm assets, production volume, } \\
\text { employment of the population and reduce the } \\
\text { likelihood of bankruptcy }\end{array}$ \\
\hline Caselli S. и др., 2019 [30] & $\begin{array}{l}38000 \text { итальянских МСП } \\
38000 \text { Italian small and medium } \\
\text { enterprises }\end{array}$ & $\begin{array}{l}\text { Наиболее эффективны гарантии для малых } \\
\text { предприятий и микропредприятий, значимое } \\
\text { повышение прибыльности отмечается для } \\
\text { производственного сектора } \\
\text { Safeguards are most effective for small businesses } \\
\text { and microenterprises, with significant gains in } \\
\text { profitability for the manufacturing sector }\end{array}$ \\
\hline $\begin{array}{l}\text { Gonzalez-Uribe J., Wang S., } \\
2020[31]\end{array}$ & $\begin{array}{l}7723 \text { английских малых фирм } \\
7723 \text { British small firms }\end{array}$ & $\begin{array}{l}\text { Гарантии позволяют удерживать ключевых } \\
\text { работников в период кризиса Guarantees } \\
\text { allow you to retain key employees during a crisis }\end{array}$ \\
\hline
\end{tabular}


Анализ данных, приведенных в таблице, показывает, что исследования проводились по МСП, гарантийным фондам и банкам, расположенным в разных странах. Кроме того, в исследованиях рассматривался широкий корпус проблем, связанных с деятельностью систем кредитных гарантий и оценки эффективности соответствующих программ. Информация, приведенная в исследованиях, которые представлены в таблице, была использована нами при определении современных концепций и принципов кредитования МСП силами финансово-кредитных организаций. Эти концепции и принципы приведены в следующих разделах настоящей статьи.

\section{Проблемы получения кредитов МСП}

Банки обычно оценивают кредитные заявки исходя из двух основных побудительных мотивов. Первый из них - защита интересов своих вкладчиков, а второй - приемлемый уровень дохода для покрытия расходов и получения прибыли. Известно, что банки уделяют много внимания залоговому обеспечению. Это логично, поскольку такое обеспечение подтверждает готовность погасить кредит. Оно помогает банкам окупить убытки в случае невозврата кредита. Эта тенденция характерна как для частных, так и для государственных банков. Кроме того, нежелание банков предоставлять кредиты МСП связано с высокими затратами на администрирование малого кредитования. Расходы на мониторинг займов, заявок на получение займов и сбор информации могут быть выше для МСП, чем для крупных фирм с передовыми технологиями и более полными данными бухгалтерского учета [32-34]. В целом можно отметить, что большинство банков в экономически развитых и в развивающихся странах имеют слабую мотивацию к предоставлению кредитов МСП по следующим основаниям:

- другие банковские продукты являются более эффективными и обеспечивают высокую доходность капитала коммерческих банков;

- жесткие нормы обеспечения надежности, ликвидности и платежеспособности, управления банковскими рисками, защиты интересов акционеров и вкладчиков часто позволяют предоставлять кредиты только крупным корпорациям или правительствам;

- отсутствие у банков достаточной информации о деятельности МСП из-за трудности получения реальной информации об имеющихся у них проблемах [35];

- сложность получения фирмами (особенно микропредприятиями) полной информации о соответствующих финансовых программах кредитования [36];

- высокий уровень предпринимательского риска и неправильная оценка своего финансового состояния предпринимателями [37];

- отсутствие необходимого залогового обеспечения, особенно для новых отраслей промышленности и сферы услуг, не имеющих материальных активов [38];

- неблагоприятный отбор МСП со стороны банков, которые хотят предоставлять кредиты заемщикам, которые имеют очень высокую доходность от инвестиций [39];

- отсутствие у большинства МСП кредитных историй, т. е. их положительной финансовой репутации и информации о погашении предыдущих кредитов.

Появление схем кредитных гарантий объясняется нежеланием банков предоставлять кредитные средства предприятиям с низким кредитным рейтингом, особенно в тех случаях, когда недостаточная кредитоспособность сочетается с отсутствием залогов [40]. В нынешних экономических условиях, когда низкая рыночная активность препятствует быстрой и успешной капитализации залогового имущества, банки еще строже подходят к обеспечению кредита. Эксперты утверждают, что заемщик, который готов предложить более высокий уровень залога, особенно личного, как дом, возможно, имеет более высокие намерения погасить основной кредит и пользуется благосклонностью банков [16]. 
Третья и четвертая причины зачастую называются в научных исследованиях асимметричной информацией. Наличие асимметричной информации приводит, как показывает исследование 50000 корпоративных кредитов в Италии, к ситуации, когда банки требуют от МСП относительно большего обеспечения, чем при кредитовании крупных предприятий [41]. Кроме того, возрастают и требования, связанные с предоставлением залога [42]. Кроме того, большинству потенциальных получателей кредитов навязываются завышенные процентные ставки.

Все это приводит к тому, что многие высокоэффективные предприятия с низким уровнем риска не берут кредиты в банках. И наоборот, займы стараются получить предприятия с низкой эффективностью и высоким риском, которые, скорее всего, не смогут погасить кредиты [43]. Возникает ситуация, в которой заемщик не является достаточно осторожным и рискует, нанося ущерб банку [44]. Недавние финансовые кризисы усугубили рассматриваемую проблему, поскольку банковские учреждения в условиях кризисных явлений практически прекратили предоставление МСП кредитов [25].

Программы кредитных гарантий компенсируют влияние вышеупомянутых факторов, поскольку они призваны устранить потери финансовых институтов в случаях невыполнения договоров займа МСП [45].

\section{Теоретические аспекты создания гарантийных фондов}

Основные концепции предоставления кредитных гарантий, а также создания систем гарантирования кредитов для субъектов малого и среднего предпринимательства приведены далее.

Кредитная гарантия - это финансовый продукт, который малый предприниматель может использовать в качестве частичной замены залога. Суть гарантии заключается в том, что выдавшая ее организация обязуется полностью или частично погасить кредит, если заемщик не выполнит договор по возврату полученных средств. В качестве гарантирующей организации в современных национальных экономиках выступают, как правило, соответствующие фонды, созданные при государственном, отраслевом или региональном участии. Гарантийные фонды стремятся облегчить доступ к долговому капиталу, предоставляя кредиторам (банкам) комфортную гарантию на значительную часть долга.

Основной целевой группой для гарантийных фондов являются средние предприятия, малые предприятия и микропредприятия, которые обладают необходимой платежеспособностью, но по каким-то причинам не могут получить банковский кредит без поддержки гарантийного фонда.

Системы кредитных гарантий состоят из трех элементов: заемщика, кредитора и гаранта. Соответственно, заемщиком является МСП, нуждающееся в финансировании. Заемщик обычно обращается за кредитом в банк, но из-за информационной асимметрии запрос на кредит банком зачастую отклоняется. В этом случае появляется необходимость в использовании гарантийных схем, основанных на привлечении гаранта. Гарантом, как уже было указано, выступает специализированный фонд [13].

Поддержка гарантийного фонда должна носить временный характер. Необходимо отметить, что если МСП после использования кредитной гарантии сумеет самостоятельно погасить свой первый кредит и обратится за вторым кредитом, то такое предприятие будет иметь кредитную биографию и сможет получить доступ к последующим кредитам уже без гарантии.

Кредитная гарантия обеспечивает снижение риска для банков, принимая на себя часть их потерь по кредитам МСП в случае отсутствия средств [46]. При этом 
гарантийный фонд оказывает свои услуги МСП на возмездной основе. Гарант безотзывно обязуется выплатить банку по первому его требованию сумму долга МСП, вплоть до максимальной гарантированной суммы (включая основную сумму долга, проценты и все другие платежи) при получении информации от банка о том, что заемщик не погасил кредит в установленный срок. Схемы кредитных гарантий различаются в зависимости от особенностей конкретных стран и отраслей [47]. При этом кредитная гарантия является финансовым продуктом, который МСП может использовать во взаимоотношениях с банками. Важно отметить, что в отличие от залога, кредитная гарантия не представляет угрозы для банка в случае не возврата кредита [32]. Обязательство гаранта полностью или частично погасить кредит в случае отсутствия средств у МСП характеризует гаранта как независимую организацию, которая выступает в качестве третьей стороны в отношениях между банком-кредитором и заемщиком [48].

Как уже отмечалось, организации, предоставляющие кредитные гарантии, в большинстве случаев финансируются из государственных источников, и таким образом программы кредитных гарантий выступают в качестве элементов государственной помощи МСП [49]. Соответственно, эти организации являются политическим инструментом снижения финансовых ограничений для МСП [50]. Снижение ограничений происходит за счет поглощения части убытков банков по кредитам, предоставленным МСП в случае их не возврата.

Гарантийные фонды имеют общую цель, заключающуюся в расширении доступа МСП к банковскому финансированию. При этом существуют различные организационно-экономические формы таких систем, обусловленные уровнем развития стран, социальными и культурными условиями, уровнем развития банковских сетей, существующими институциональными нормами, а также стоящими задачами по развитию предпринимательского сектора экономики. Нельзя утверждать, что существует одна идеальная модель кредитных гарантий. В конкретных условиях необходимо обосновывать эффективность создания наилучшего типа гарантийного фонда для конкретного контекста.

В настоящее время сформировались 16 основных принципов создания систем кредитных гарантий $[5,10,15,16,24]$ :

- гарантийный фонд должен быть независимым юридическим лицом, действующим на основе четко определенной нормативно-правовой базы;

- программа кредитных гарантий должна иметь адекватное финансирование для достижения своих политических целей, при этом источники финансирования должны быть прозрачными;

- законодательная и нормативная база, регулирующая работу фонда, должна обеспечивать справедливое отношение ко всем участникам системы гарантирования;

- гарантийный фонд должен быть под эффективным надзором;

- система гарантий должна строить свою деятельность на основе утвержденной стратегии и оперативных целей;

- гарантийный фонд должен иметь надежную структуру управления с компетентным советом директоров, назначаемым в соответствии с четко определенными критериями;

- работа фонда должна основываться на внутреннем контроле деятельности;

- в процессе деятельности фонда должны постоянно оцениваться возникающие риски;

- гарантийный фонд должен иметь четко определенные критерии отбора для МСП, банков и их кредитных инструментов; 
- при предоставлении гарантий необходимо исходить из компромисса между информационно-пропагандистской деятельностью и финансовой устойчивостью;

- как правило, выдаваемые гарантии должны быть частичными, т. е. обеспечивающими правильные стимулы для МСП и банков;

- стоимость услуг, предоставляемых МСП гарантийным фондом, должна основываться на имеющихся рисках, чтобы сохранять финансовую устойчивость фонда;

- процесс управления претензиями должен быть эффективным и обеспечивать стимулы для возмещения убытков по кредитам;

- гарантийные фонды должны подчиняться строгим требованиям к финансовой отчетности и проверяться с использованием внешнего аудита;

- фондам необходимо периодически раскрывать информацию, связанную с их деятельностью;

- государственные органы должны оценивать деятельность созданных ими фондов.

Экономическое и финансовое воздействие систем кредитных гарантий, как показывает опыт $[4,51]$, позволяет дополнительному количеству МСП получить доступ к банковскому финансированию, а также широкий доступ к льготным финансовым условиям, а именно большим суммам кредитов и более длительным срокам их погашения. Поддержка деятельности МСП, исключенных из традиционного кредитования, может привести к привлечению новых предпринимателей и снижению безработицы. Кроме того, наиболее остро проблема кредитования стоит в относительно слаборазвитых регионах, поэтому кредитные гарантии могут положительно влиять на развитие МСП в них.

\section{Особенности деятельности современных систем кредитных гарантий}

В настоящее время в разных странах действуют самые разнообразные гарантийные фонды. Основным элементом, который отличает гарантийные программы от других государственных мер поддержки МСП, таких как субсидии или гранты, заключаются в том, что гарантийные фонды используют банки для определения получателей гарантированных кредитов.

Представляет интерес деятельность ряда систем кредитных гарантий. В Европейском Союзе в последние годы были реализованы четыре программы таких гарантий для MCП [29]. Программа «GEI» была направлена на поддержку занятости и охватывала 1998-2000 гг. Гарантированная сумма кредитов по ней составила 2,4 млрд евро. Общий объем поддерживаемых кредитов составил 6,17 млрд евро. При этом затраты на выполнение программы (включая потери от невозвратных кредитов) составили всего 0,14 млрд евро (ЕСA, 2017). Вторая программа - «SMEG 2001»- осуществлялась в 2001-2006 гг. Общий объем поддерживаемых кредитов составил 10,22 млрд евро, при этом были гарантированы кредиты на 4,68 млрд евро. Стоимость реализации программы составила 0,27 млрд евро. Третья программа - «CIP SMEG»-была нацелена на развитие конкурентоспособности и инноваций МСП и выполнялась в 2007-2013 гг. Общий объем поддерживаемых кредитов составил 14,4 млрд евро, при этом были выданы гарантии на 7,34 млрд евро. Затраты на выполнение программы (включая потери по кредитам) составили 0,64 млрд евро. С 2014 г. началась четвертая программа, нацеленная на повышения конкурентоспособности МСП, - «COSME», ее планируется завершить в 2020 г. Благодаря этой программе по состоянию на начало 2019 г. более 414 тысяч МСП уже воспользовались улучшенными условиями финансирования. Ожидаемая гарантированная сумма составит 7,31 млрд евро, планируется поддержать кредиты в размере 14,89 млрд евро. Стоимость реализации программы с учетом потерь ожидается в размере 0,72 млрд евро за весь период с 2014 по 2020 г. 
В США действует несколько программ кредитных гарантий, наиболее важной из которых является программа «7(a) Loan Program», которое управляется администрацией малого бизнеса (SBA). Гарантированные кредиты предоставляются для финансирования различных целей, в том числе пополнения оборотного капитала, инвестиций в основной капитал и землю, а также при особых условиях для покрытия задолженности. Важно отметить, что для получения права заемщики должны подтвердить, что они не смогли получить кредиты на обычном финансовом рынке. Коэффициент покрытия зависит от объема кредита. В случае кредитов 150 тысяч долларов гарантируются до 85 \%. Максимальная сумма кредита составляет 2 миллиона долларов. Срок погашения зависит от использования кредита. Для оборотного капитала порог составляет 10 лет. Гарантийные сборы выражаются в процентах от суммы гарантии и обычно оплачиваются заемщиком. Они состоят из авансового взноса и ежегодного взноса. Последний зафиксирован на уровне $0,54 \%$.

В Японии [35] кредитные гарантии финансируются Министерством финансов через Министерство экономики, торговли и промышленности. Непосредственно гарантированием кредитов занимается Японская федерация корпораций кредитных гарантий (Japan Federation of Credit Guarantee Corporations). Эта федерация включает 51 корпорацию кредитных гарантий (Credit Guarantee Corporations), по одной в каждой префектуре, а также в городах Кавасаки, Гифу, Нагоя и Иокогама. Каждая из них работает непосредственно с МСП и банками, выдающими кредиты. Правительство также предоставляет средства для страхования выданных с гарантией кредитов Японской финансовой корпорации (Japan Finance Corporation). В 2013 г. были гарантированы кредиты для 1,46 миллиона МСП, что составляло почти $40 \%$ от общего количества этих предприятий в стране. Совокупный объем гарантированных кредитов составил около 30 трлн иен.

Поскольку банки имеют определенный опыт в оценке кредитоспособности заемщиков, то гарантийные фонды могут использовать этот опыт, чтобы помочь избежать плохих заемщиков. В то же время некоторые фонды в США сами занимаются проверкой кредитоспособности МСП. Хотя такая оценка увеличивает расходы гарантийного фонда, она считается оправданной, если фонд обладает информационным преимуществом, которое позволяет ему проводить более качественную проверку и тем самым снизить уровень невозврата кредитов [51]. Такой подход, безусловно, логичен при наличии соответствующего информационного преимущества по сравнению с банковскими структурами, не всегда располагающими доступом к соответствующей информации. Вместе с тем этот подход целесообразен при выдаче значительных кредитных сумм. В случае небольших кредитов соответствующие проверки могут стать сопоставимыми по стоимости с суммой гарантии.

Существование государственных гарантий может привести к двум видам последствий. С одной стороны, это может снизить рыночную дисциплину, поскольку МСП знают, что их кредиты будут обеспечены правительством, и таким образом привести к проблемам с возвратом кредитов. Как уже указывалось, для борьбы с таким явлением целесообразно гарантировать не всю сумму кредита, а только ее часть. В качестве примера можно привести опыт Японии, где в конце XX в. имели место полные гарантийные схемы, которые покрывали 100 \% выданного кредита [52]. Отрицательный опыт такого подхода, когда уровень невозвратных кредитов достигал 7,3 \% [15], вынудил Японскую федерацию корпораций кредитных гарантий (о которой упоминалось выше) в настоящее время выдавать гарантии не более чем на 80 \% суммы кредита. Для американской программы «7(a) loan program» в случае кредитов до 150 тысяч долларов максимальная гарантия достигает $85 \%$. По программам гарантирования кредитов в Европейском Союзе, 
описанным выше, средние значения гарантий были на уровне $50 \%$ от суммы кредитов [29]. Необходимо отметить, что существует взаимная связь между прозрачностью деятельности МСП и процентом гарантирования кредита. В разных странах Европейского Союза наибольшие значения гарантий не превышают 80 \% для МСП, деятельность которых может полностью контролироваться банками. В этих же странах МСП, которые не готовы в полной мере обеспечивать контроль над своей деятельностью, могут рассчитывать на уровень гарантии 20-30\%.

Потери государства от принятия на себя риска могут компенсироваться за счет роста объемов производства, налоговых платежей, снижения безработицы и решения других социально-экономических задач [22]. Выгоды и издержки, понесенные в результате существования программы кредитных гарантий, могут быть измерены через сравнение не возвращенных сумм кредитов с экономическим эффектом, полученным за счет выдачи дополнительных кредитов в связи с существованием схем гарантирования.

При создании гарантийных фондов в каждом конкретном случае необходимо оценивать три аспекта их деятельности. Во-первых, каков оптимальный коэффициент кредитных гарантий (т. е. какая доля кредита должна покрываться гарантией) для достижения поставленной цели минимизации невозвратных кредитов МСП и в то же время обеспечения поддержки МСП. Во-вторых, должно ли быть это соотношение постоянным для различных отраслей и регионов независимо от макроэкономических условий. В-третьих, должна ли ставка быть постоянной для всех банков или она должна варьироваться в зависимости от финансовой устойчивости банка. Понятно, что организаторы фондов должны снижать гарантийный коэффициент в хороших экономических условиях и повышать его в условиях кризисных явлений.

Известно, что фонды кредитных гарантий работают на основе возмещения затрат, а некоторые даже приносят прибыль. Процентные ставки для заемщиков гарантийного фонда определяются различными способами. Важной для них является стоимость услуг систем кредитных гарантий. Большинство действующих в настоящее время гарантийных фондов ограничивают размер этих платежей. Например, в Таиланде гарантийный фонд установил плату за свои услуги в размере примерно $1 \%$ [53]. Отметим, что в английской программе «The Enterprise Finance Guarantee (EFG)» за выдачу гарантии по кредиту необходимо заплатить $2 \%$ от суммы кредита [31]. В большинстве стран услуги систем кредитных гарантий находятся в пределах от 1 до $3 \%$ [9, 14, 21]. Вместе с тем необходимо отметить, что, как правило, использование услуг гарантийного фонда существенно меньше по сравнению с высокопроцентными кредитами без гарантий или финансированием на сером кредитном рынке.

Основное преимущество гарантийных фондов заключается в том, что они могут использовать свой капитал с большим эффектом. Это связано с тем, что такие фонды не выдают кредиты, а только обеспечивают гарантии под имеющиеся средства. Другими словами, общий объем гарантированных кредитов может в 5-10 раз превышать капитал самого фонда. Опыт Японии и Германии свидетельствует о том, что после 10 лет работы гарантийные фонды могут увеличить кратность до 20 и более $[5,15]$. По мере погашения кредитов заработанные средства увеличивают объем доступного капитала. Однако этот мощный мультипликатор - лишь одно из преимуществ гарантийных фондов. Еще одним преимуществом является то, что налаживание отношений с банками позволит не только повысить шансы МСП на получение будущих кредитов, но и создать кредитную историю для других кредиторов.

Создание гарантийного фонда начинается с первоначального вложения капитала. Этот капитал предоставляется, как правило, органами власти (от городского уровня до 
национального уровня). Затем гарантийный фонд заключает партнерские соглашения с банками, в которых излагаются конкретные оперативные мероприятия. Эти соглашения могут варьироваться в зависимости от банков-партнеров, но обычно включают такие положения, как типы заемщиков, имеющих право на получение гарантий, размер, срок и назначение займов, а также механизм выплаты гарантий.

Несмотря на существование различных моделей гарантийных фондов, этапы получения МСП гарантий по кредитам типичны:

- заемщик сначала обращается непосредственно в банк за кредитом;

- банк рассматривает заявку и принимает решение одобрить или отклонить ее;

- если заявка будет одобрена, то кредит будет предоставлен, а гарантийный фонд не будет принимать в этом участие;

- если кредит не одобрен из-за того, что у МСП отсутствует необходимый уровень залога, но потенциальный заемщик соответствует критериям, установленным договором между банком и гарантийным фондом, то заявка будет передана в гарантийный фонд;

- заявка на кредит будет рассмотрена гарантийным фондом и по ней будет принято решение об одобрении или отказе;

- в случае, когда кредитная заявка одобрена гарантийным фондом, он предоставляет банку соответствующую гарантию;

- если заемщик погашает кредит, то гарантийный фонд освобождается от выданной гарантии;

- если заемщик не выполняет свои обязательства по кредиту, а банк выполняет все свои обязательства в полном объеме пытаясь взыскать долг, то гарантийный фонд должен возместить банку сумму гарантии.

\section{Заключение}

В статье предпринята попытка обобщить некоторые предыдущие научные исследования, посвященные роли и влиянию систем кредитных гарантий в снижении ограничений на банковское финансирование для малых и средних предприятий. Рассматриваются основные принципы и концепции реализации гарантийных программ, осуществляемых с государственным участием. При этом показано, что кредитные гарантии являются одним из инструментов, широко используемых при поддержке предпринимательского сектора экономики. Системы кредитных гарантий обеспечивают повышение устойчивости национальных экономик, поскольку оказывают положительное влияние не только на деятельность МСП, но и на функционирование финансово-кредитных организаций и решают ряд социально-экономических вопросов.

Проведенный анализ выявил ряд факторов, способствующих успеху программ кредитных гарантий. При этом кредитные гарантии будут успешными, когда МСП хорошо подготовлены к обеспечению прозрачной эффективной деятельности, направленной на безусловный возврат выданных им гарантированных кредитов. В случаях, если имеются трудности в контроле над деятельностью МСП, эти МСП не могут рассчитывать на высокий уровень гарантий. Совместная работа гарантийных фондов и банков должна быть направлена на решение имеющихся в настоящее время проблем слабого финансирования предпринимательского сектора, характерного для развитых и развивающихся стран.

В настоящее время системы гарантирования кредитов МСП получили значительное развитие в большинстве стран с рыночной экономикой. Необходимость в них обусловлена, как показал приведенный в статье анализ, объективными сложностями в предоставлении банковских кредитов средним и особенно малым предприятиям. Проведенные к настоящему времени исследования показали, что только государственные 
программы кредитных гарантий способны в полной мере решить проблему финансирования МСП. При этом наиболее целесообразным является выдача гарантий, обеспечивающих частичный объем кредитования, когда заемщики также несут определенную ответственность по возврату полученных средств. Уровень гарантирования кредитов в зависимости от конкретных условий, а также стран, в которых расположены МСП, имеет значительную дифференциацию в пределах от 20 до $85 \%$.

Существенным представляется модификация схем предоставления гарантийных кредитов в зависимости от видов деятельности конкретных предприятий, а также от предпринимательского климата, характерного для конкретных регионов.

Полученные результаты имеют теоретическое и практическое значение. Они могут быть использованы в деятельности гарантийных организаций, финансово-кредитных учреждений, а также государственных структур и общественных организаций, при решении задач оказания поддержки предпринимателям. Итоги анализа представляют интерес для руководителей и работников малых и средних предприятий.

Полученные данные могут быть использованы в образовательных процессах высших и средних учебных заведений.

Дальнейшие исследования должны, на наш взгляд, проводятся с учетом особенностей муниципальных образований, в которых расположены МСП, а также социальных аспектов их деятельности (женские предприятия, молодежное предпринимательство, социальные предприятия).

\section{СПИСОК ЛИТЕРАТУРЫ REFERENCES}

1. Acs Z.J., Estrin S., Mickiewicz T.M., Szerb L. Entrepreneurship, institutional economics, and economic growth: an ecosystem perspective. Small Business Economics, 2018, vol. 51, pp. 501-514.

2. Janković G., Golubović M. Open innovation in small and medium-sized enterprises. Business, 2019 , vol. 65(3), pp. 89-101.

3. Rotar L.J., Pamic R.K., Bojnec S. Contributions of small and medium enterprises to employment in the European Union countries. Economic Research-Ekonomska Istraživanja, 2019, vol. 32, no. 1, pp. 3302-3314.

4. Kraemer-Eis H., Botsari A., Lang F., Torfs W., Gvetadze S. European Small Business Finance Outlook. EIF Research \& Market Analysis. Working Paper 2018/53. European Investment Fund, 2018. 134 p. Available at: https://www.eif.org/news_centre/publications/EIF_Working_Paper_2018_53.pdf (accessed 11 May 2020).

5. Asia SME Finance Monitor 2014. Manila, Asian Development Bank, 2015. 304 p. Available at: https://www. adb.org/sites/default/files/publication/173205/asia-sme-finance-monitor2014.pdf (accessed 11 May 2020).

6. Narteh B. SME bank selection and patronage behaviour in the Ghanaian banking industry. Management Research Review, 2013, vol. 36 (11), pp. 1061-1080.

7. Beck T., Klapper L.F., Mendoza J.C. The typology of partial credit guarantee funds around the world. Journal of Financial Stability, 2010, vol. 6 (1), pp. 10-25.

8. Kraemer-Eis H., Lang F., Torfs W., Gvetadze S. European Small Business Finance Outlook. Working Paper 2015/32. EIF Research \& Market Analysis. Luxembourg, 2015. 97 p. Available at: https://www.ggb.gr/ sites/default/files/basic-page-files/EIF_2015\%20European\%20Small\%20Business\%20Finance\%20Outlook_ 2015_32.pdf (accessed 11 May 2020).

9. Zander R., Miller C., Mhlanga N. Credit Guarantee Systems for Agriculture and Rural Enterprise Development. Rome, Food and Agriculture Organization of the United Nations, 2013. 109 p. Available at: http://www.fao.org/3/i3123e/i3123e00.pdf (accessed 11 May 2020).

10. Nitani M., Riding A. Promoting enterprise development or subsidizing tradition? The Japan credit supplementation system. International Small Business Journal, 2005, no. 23, pp. 48-71.

11. The SME Financing Gap: Theory and Evidence. Financial Market Trends, 2006, vol. 2006, Iss. 2, pp. 89-97. Available at: http://econpapers.repec.org/article/oecdafkab/519gd5ngghjg.htm (accessed 11 May 2020).

12. Llisteri J.J. Credit guarantee schemes: preliminary conclusions. Financier, 1997, no. 4. pp. 95-112.

13. Riding A.L., Haines G.Jr. Loan guarantees: costs of default and benefits to small firms. Journal of Business Venturing, 2001, vol. 16 (6), pp. 595-612. 
14. Gozzi H.C., Schmuckler S. Public credit guarantees and access to finance. 2016. Available at: https://www2.warwick.ac.uk/fac/soc/economics/research/workingpapers/2016/twerp_1122_gozzi.pdf (accessed 11 May 2020).

15. SME and entrepreneurship financing: the role of credit guarantee schemes and mutual guarantee societies in supporting finance for small and medium-sized enterprises. Final report of the WPSMEE. OECD Publishing. 2013. Available at: http://www.oecd.org/officialdocuments/publicdisplaydocumentpdf/?cote= CFE/SME(2012)1/FINAL \&docLanguage=En (accessed 11 May 2020).

16. Deelen L., Molenaar K. Guarantee funds for small enterprises: a manual for guarantee fund managers. International Labor Organization, 2004. 140 p. Available at: https://www.ilo.org/global/publications/ilobookstore/order-online/books/WCMS_PUBL_9221160335_EN/lang--en/index.htm (accessed 11 May 2020).

17. Riding A., Madill J., Haines G.Jr. Incrementality of SME Loan Guarantees. Small Business Economics, 2007, vol. 29. pp. 47-61.

18. Beck T., Klapper L.F., Mendoza J.C. The typology of partial credit guarantee funds around the world. Journal of Financial Stability, 2008, vol. 6(1), pp. 10-25.

19. Posey R., Reichert A. Terms of lending for small business lines of credit: the role of loan guarantees. The International Journal of Business and Finance Research, 2011, vol. 59 (1), pp. 91-102.

20. Saadani Y., Arvai Z., Rocha R. A review of credit guarantee schemes in Middle East and North Africa Region. The World Bank, Middle East and North Africa Region, Financial and Private Sector Development Unit, 2011. 40 p. Available at: https://documents.worldbank.org/en/publication/documents-reports/documentdetail/201881468050950823/a-review-of-credit-guarantee-schemes-in-the-middle-east-and-north-africa-region (accessed 21 May 2020).

21. Leone P., Panetta I.C., Porretta P. Credit guarantee institutions, performance and risk analysis: an experimental scoring. Bank Stability, Sovereign Debt and Derivatives. Palgrave Macmillan Studies in Banking and Financial Institutions. Ed. by Falzon J. London, Palgrave Macmillan, 2013. pp. 115-160.

22. Gropp R., Gruendl C., Guettler A. The impact of public guarantees on bank risk-taking: evidence from a natural experiment. Review of Finance, 2013, vol. 18 (2), pp. 457-488.

23. Anginer D., De la Torre A., Ize A. Risk-bearing by the state: When is it good public policy? Journal of Financial Stability, 2014, vol. 10, pp. 76-86.

24. Principles for public credit guarantee schemes for SMEs. Task force for the design, implementation and evaluation of public credit guarantee schemes for small and medium enterprises. Washington D.C., The World Bank and FIRST Initiative, USA, 2015. 64 p.

25. Chatzouz M., Gereben A., Lang F., Torfs W. Credit guarantee schemes for SME lending in Western Europe. European Investment Fund Working Paper 2017/42. Luxembourg, 2017. 73 p. Available at: https://www.econstor.eu/bitstream/10419/176672/1/eif-wp-42.pdf (accessed 21 May 2020).

26. Liang L.W., Huang B.Y., Liao C.F., Gao Y.T. The impact of SMEs' lending and credit guarantee on bank efficiency in South Korea. Review of Development Finance, 2017, vol. 7 (2), pp. 134-141.

27. Balog A. Analysis of SME segment lending processes in light of credit guarantees in the European Union. Financial and Economic Review, 2018, vol. 17, no. 1, pp. 62-82.

28. Saito K., Tsuruta D. Information asymmetry in small and medium enterprise credit guarantee schemes: evidence from Japan. Applied Economics, 2018, vol. 50, pp. 2469-2485.

29. Brault J., Signore S. The real effects of EU loan guarantee schemes for SMEs: a pan-European assessment. Working Paper 2019/56. Luxembourg, European Investment Fund, 2019. 45 p. Available at: https://www.eif.org/news_centre/publications/EIF_Working_Paper_2019_56.pdf (accessed 21 May 2020).

30. Caselli S., Corbetta G., Rossolini M., Vecchi V. Public credit guarantee schemes and SMEs' profitability: evidence from Italy. Journal of Small Business Management, 2019, vol. 57, pp. 555-578.

31. Gonzalez-Uribe J., Su Wang. The effects of small-firm loan guarantees in the UK: insights for the COVID-19 pandemic crisis. London School of Economics, University of Amsterdam, 2020. 60 p. Available at: https://juanitagonzalez-uribe.net/wp-content/uploads/2020/05/Gonzalez_Uribe_Wang_13052020.pdf (accessed 21 May 2020).

32. Green A. Credit guarantee schemes for small enterprises: an effective instrument to promote private sectorled growth? UNIDO, 2003. Available at: https://www.unido.org/sites/default/files/2007-11/18223_PSDseries10_0.pdf (accessed 21 May 2020).

33. Credit guarantee schemes for SME lending in Central, Eastern and South-Eastern Europe. Vienna Initiative Working Group on Credit Guarantee Schemes. 2014. Available at: http://vienna-initiative.com/wp-content/uploads/2014/11/2014_credit_guarantee_schemes_report_en.pdf (accessed 11 May 2020).

34. Brown J.D., Earle J.S. Finance and growth at the firm level: evidence from SBA loans. The Journal of Finance, 2017, vol. 72, pp. 1039-1080. 
35. Yoshino N., Taghizadeh-Hesary F. Optimal credit guarantee ratio for Asia. ADBI Working Paper 586. Tokyo, Asian Development Bank Institute, 2016. 25 p. Available at: https://www.adb.org/sites/default/files/publication/189374/adbi-wp586.pdf (accessed 21 May 2020).

36. Tunahan H., Dizkirici A.S. Evaluating the Credit Guarantee Fund (KGF) of Turkey as a Partial Guarantee Program in the Light of International Practices. International Journal of Business and Social Science, 2012, vol. 3(10), pp. 79-92.

37. Pinkovetskaia I.S., Sverdlikova E.A., Lebedev A.V. Estimating entrepreneurial risk in Russian small and medium-sized enterprises. The Journal of Contemporary Issues in Business and Government, 2019, vol. 25 (1), pp. 30-43.

38. Samujh R.-H., Twiname L., Reutemann J. Credit guarantee schemes supporting small enterprise development: a review. Asian Journal of Business and Accounting, 2012, vol. 5 (2), pp. 21-40.

39. Moro A., Lucas M.R., Kodwani D. Trust and the demand for personal collateral in SME-bank relationships. Journal of Entrepreneurial Finance, 2012, vol. 16, pp. 57-79.

40. Hull J.C., Predescu M., White A. Bond prices, default probabilities and risk premiums. 2005. Available at: https://ssrn.com/abstract=2173148 (accessed 11 May 2020).

41. Pozzolo A.F. The role of guarantees in bank lending. EFMA 2004 Basel Meeting Papers, 2004. 24 p. Available at: https://doi.org/10.2139/ssrn.498982 (accessed 11 May 2020).

42. Baas T., Schrooten M. Relationship banking and SMEs: a theoretical analysis. Small Business Economics, 2006, vol. 27 (2-3), pp. 127-137.

43. Nicholson W., Snyder C. Microeconomic theory: basic principles and extensions. USA, Thomson Higher Education Mason, 2008. 742 p. Available at: https://edisciplinas.usp.br/pluginfile.php/2871502/ mod_resource/content/1/Nicholson\%20\%20Snyder\%20\%282007\%29\%20-\%20Microeconomic\%20theory \%20\%2010\%20ed..pdf (accessed 11 May 2020).

44. Rowell D., Connelly L.B. A history of the term «moral hazard». Journal of Risk and Insurance, 2012, vol. 79 (4), pp. 1051-1075.

45. Craig B.R., Jackson W.E., Thomson J.B. Credit market failure intervention: do government sponsored small business credit programs enrich poorer areas? Small Business Economics, 2008, vol. 30, pp. 345-360.

46. Levitsky J. Credit guarantee schemes for SMEs - an international review. Small Enterprise Development, 1997, vol. 8 (2), pp. 4-17.

47. Beck T., Klapper L.F., Mendoza H.C. The typology of partial credit guarantee funds around the world. Journal of Financial Stability, 2010, vol. 6 (1), pp. 10-25.

48. O’Bryan W.E. An analysis of small business loan guarantee funds. University of Nebraska-Lincoln. 2010. Available at: http://digitalcommons.unl.edu/cgi/viewcontent.cgi?article= 1003\&context=arch_crp_theses (accessed 11 May 2020).

49. Douette A., Lesaffre D., Siebeke R. SMEs' credit guarantee schemes in developing and emerging economies: reflections, setting-up principles, quality standards. Deutsche Gesellschaft für Internationale Zusammenarbeit, 2013. 141 p. Available at: http://www.ruralfinanceandinvestment.org/sites/default/files/giz-studyon-smes-credit-guarantee-schemes_pdf.pdf (accessed 11 May 2020).

50. Holton S., McCann F., Prendergast K., Purdue D. Policy measures to improve access to credit for SMEs: a survey. Quarterly Bulletin Articles, 2013, pp. 91-110. Available at: https://ideas.repec.org/a/cbi/qtbart/y2013m10p91-110.html (accessed 11 May 2020).

51. Honohan P. Partial credit guarantees: principles and practice. Journal of Financial Stability, 2010, vol. 6 (1), pp. 1-9.

52. Uesugi I., Sakai K., Yamashiro G.M. Effectiveness of Credit Guarantees in the Japanese Loan Market. RIETI Discussion Paper Series. Tokyo, The Research Institute of Economy, Trade and Industry, 2006. 25 p.

53. Anuchitworawong C., Intarachote T., Vichyanond P. The economic impact of small business credit guarantee. TDRI Quarterly Review, 2006, vol. 21 (6), pp. 17-21.

Поступила 05.07.2020 г. 
UDC 331.5

\title{
CREDIT GUARANTEES FOR SMALL AND MEDIUM ENTERPRISES: FOREIGN EXPERIENCE
}

\author{
Iuliia S. Pinkovetskaia, \\ judy54@yandex.ru \\ Ulyanovsk State University, \\ 42, L. Tolstoy street, Ulyanovsk, 432000, Russia
}

Iuliia S. Pinkovetskaia, Cand. Ec. Sc., associate professor, Ulyanovsk State University.

The relevance of the research is caused by the fact that small and medium entrepreneurship play an important role in the national economies of the absolute majority of both developed and developing countries. At the same time, one of the significant barriers to its further growth is weak lending by banks to the business sector. One of the main ways to overcome this situation is to develop and implement loan guarantee programs. The purpose of the research is to study the experience of lending to small and medium enterprises using guarantee funds, to analyze the principles and concepts of creating credit guarantee systems. The problems of issuing Bank loans to small and medium businesses were considered, the international experience of implementing guarantee lending programs was studied, and the existing schemes for organizing this activity were considered. The research methodology is based on an analysis of the activities of national, regional and industry guarantee funds, as well as financial and credit organizations that specialize in issuing guarantees for small and medium enterprises. At the same time, the main attention is paid to state guarantees of lending to such enterprises. The experience of credit guarantee systems in Europe, North and Latin America, Asia and Africa was reviewed. The results showed that loan guarantee programs play a major role in reducing restrictions on Bank financing for small and medium enterprises. The article presents the basic principles and concepts of guarantee programs implemented with state participation. It is shown that credit guarantees are one of the tools widely used in support of the business sector of the economy. At the same time, it is most appropriate to issue guarantees that provide a partial volume of lending, when borrowers also bear some responsibility for the return of funds received. The results obtained can be used in the activities of guarantee organizations, financial and credit institutions, and state authorities. The results of the analysis are of interest to managers and employees of small and medium enterprises.

Key words: Small and medium enterprises, guarantee funds, banks, credit guarantee systems, entrepreneurship financing.

Received: 5 July 2020. 